\title{
Gaining Cultural Intelligence through Second Life Learning Interventions
}

\author{
doi:10.3991/ijac.v3i3.1351 \\ Sarah E. Siegel \\ IBM Center for Advanced Learning, Armonk, New York, USA \\ Columbia University, New York, USA
}

\begin{abstract}
Companies' investment in international assignments remains essential, but is affordable for only relatively few employees. In today's global economy, many employees must gain cultural intelligence. Encyclopedic web sites and smart game simulations are ill-equipped to offer the live, authentic immersive experience of Virtual World environments; learning from colleagues who are country-natives through interacting with them in a Virtual World like Second Life is the next best thing to being there, and it requires no travel. Virtual world learning sessions with local ambassadors are affordable on a massive scale compared with international assignments. In addition, through Virtual World experiences, international assignees can be more effective with colleagues from their assigned countries prior to boarding planes, even prior to Day 1 of the assignment. This research proposal, including a literature review and a research design, asks, and begins to answer: What can cultural intelligence learning in Second Life achieve or advance that cultural intelligence learning in real-life within the cultures, smart game simulations and encyclopedic, self-service web sites cannot as readily?
\end{abstract}

Index Terms-CQ, cultural intelligence, Second Life, Virtual World-based learning.

\section{INTRODUCTION}

Increasingly, IBM employment worldwide depends on cultural intelligence; India's and other growth markets', e.g., China's and Eastern Europe's, continue to increase revenue and employee populations faster than IBM is growing today in the United States and other major markets, e.g., Germany and Japan. Corporate employment altogether depends on cultural intelligence; IBM's experience is not unique, however, it is among the biggest global companies, and so is qualified to help its own employees gain cultural intelligence, as well as to help its largest, global clients do so.

Today, companies have an unprecedented opportunity, thanks to Virtual World technology. The technology encourages cultural intelligence-building through immersive experiences with colleagues from around the world and requires no travel. The businesspeople who are succeeding in today's society, and increasingly in tomorrow's, are those with the most cultural intelligence, i.e., who can; are willing to; and who do adapt their communication, negotiation and leadership styles, according to the cultures with which they are dealing, and who can overcome crosscultural challenges to achieve the business' desired outcomes. Arguably, they tend, also, to be the people, who are open to new technology, e.g., Virtual World environments to help them with their cross-cultural goals.

\section{LITERATURE REVIEW}

This literature review analyzes a number of works related to cultural intelligence and to learning in virtual world environments. Ultimately, my research aim is to demonstrate that businesspeople can gain a degree of cultural intelligence through experiential learning in a virtual world such as Second Life - that it can be the next best thing to being there, and in a number of cases, even more effective than being there initially.

“Culturally intelligent” people are, “...individuals who are extraordinarily effective in getting along with people from other cultures...(they have high CQ)" [1]. While an increasingly robust body of literature is being written on the value of cultural intelligence, “...little is known about the processes through which CQ is developed..." [2]. Since "cultural intelligence," as defined above, is about cross-cultural effectiveness [1] - and not defined strictly as a classic sort of intelligence, i.e., not exclusively an innate attribute - cultural intelligence, to a degree, should be able to be learned and developed over time.

Understandably, there is no better way to develop deep cultural intelligence about a particular culture than to immerse oneself by living and working in it; people who go abroad have "...higher levels of cultural intelligence" [3]. No wonder relatively little is written on how one learns and gains cultural intelligence, since historically, the world-class way has been via direct experience in the cultures per se.

Businesspeople can benefit more than ever from demonstrating cultural intelligence in today's global economy, since businesspeople at all levels are working with clients and colleagues from other countries to an unprecedented degree [4].

Do firms that focus on encouraging cultural intelligence perform better than those that do not? Gelfand, Imai, and Fehr [2] wonder: "Are there 'culturally intelligent organizations' that, through their values, assumptions, policies, and procedures, create cultural adaptation at the organizational level?” And do firms that attract culturally intelligent applicants have an easier time of identifying willing international assignees? [2]. At a more macro level as well, Gelfand, Imai, and Fehr [2] seem to ask rhetorically, "In this era of globalization, are there societal advantages, such as higher economic outcomes and lower international conflict in countries with high levels of CQ?” 


\section{A. Why and How to Manage Cross-cultural Teams}

Even with the business and societal advantages that cultural intelligence should deliver, and the high volume of businesspeople who need to gain cultural intelligence, still, large businesses cannot afford physical immersion for all of them. In global companies, Adler and Gunderson [4] argue, "...everyone from the CEO to the lowest-level worker must use cross-cultural skills.” The research [4] concludes that since most cross-cultural teams' diversity is:

...more frequently ignored than well managed...culturally diverse teams often perform below expectations and below organizational norms....Diversity becomes most valuable when the need for the team to reach agreement (cohesion) remains low relative to the need to invent creative solutions.

To enable innovation, the research [4] includes guidelines for maximizing integration and productivity among cross-cultural teams; the guidelines, however, do not include advocacy for any sort of technology. In addition, there is no suggestion on how to train leaders even as Adler and Gunderson [4] cite Fiedler, Meuwese, \& Oonk [5]: "Research indicates that 'culturally trained leader[s], regardless of leadership style... achieve... high[er] levels of performance and rapport than do non-trained leaders.”

\section{B. Cultural Intelligence Gained by Academic or Business Sojourns Abroad}

Focusing on cultural intelligence antecedents, Crowne's [3] survey of 140 people concludes that spending time abroad - whether for school or work - increases cultural intelligence. Crowne [3] also recommends conducting cultural intelligence training in foreign countries, whether for employees going on international assignments or for anyone interacting with people from beyond their countries at work. By contrast, the work [3] cautions readers against relying only on technology to interact with colleagues abroad.

The journal article's [3] copyright surprises me; with the amount of employees requiring cultural intelligence in this era, does Crowne [3] really think that her travel recommendation is broadly affordable? Also, the study [3] suggests that by using technology, people will miss cues via “...body language and tone;" however, virtual world environments, e.g., Second Life, enable social presence, gestures and even real-time conversations, using participants' own voices, i.e., tones through VoIP. Primarily, Crowne [3] asserts implications for business, and so I would expect the vast majority of respondents to be businesspeople, rather than students. That is another weakness in the sampling data, as they are U.S.-centric and studentfocused; fully $89 \%$ of the survey respondents are American; $85 \%$ of them are students; and 64\% are currently employed while being part-time students.

Also, there is no control group [3], which threatens internal validity. Finally, there is no explanation of the correlation between respondents' self-reports of time abroad for school or work and the corresponding cultural intelligence assessment as determined by the Ang and Van Dyne [6] survey that the researcher [3] cites.

\section{Action Learning in Second Life}

Wagner and Ip [7] write that action learning has been widely used in leadership development training and, “...focuses on action but also includes exploration (planning) and reflection (experience and understanding).” The researchers [7] conduct an action learning pilot in Second Life to enable senior-level undergraduates to gain practical business experience without risking missteps among established, real-world firms; the students create their own businesses and are surveyed after receiving their grades on whether they feel the assignment represents action learning, and whether the learning experience is worth the heavy work-load involved in completing it.

This [7] has the makings of an excellent research study, and yet some fundamental elements seem wrong to me: the research participants, the research questions, or both. For generalizability's sake, it seems like the perfect pilot to conduct with undergraduate Business majors, rather than Management Information Systems (MIS) students. The researchers [7] are Information Systems department members; they determine to use MIS students, and to instill in them the discipline involved in the formal steps of action learning, but the students disappoint the researchers. The students recognize that they are doing action learning, but are not conscious of the formal steps [7]. In the future, I would recommend using Business students for the pilot and interviewing them instead about the business best practices that are reinforced by the experience and the implications for their lessons learned had they made the same mistakes in real-world businesses. Action learning could then be inferred and identified by the researchers, without necessarily making the students conscious of it.

\section{Cross-cultural Training Games vs. Real-time, Virtual World-based Experiences:}

Upon completing on an online "Super Search" of the Gottesman Library of Teachers College, I conclude that there is not yet explicit literature on using real-time, virtual world technology intentionally to help people increase their cultural intelligence. There is, however, research sponsored by the U.S. Army, including Taylor's and Sims' [8] on the value of creating smart agents and, effectively, online games for cross-cultural training. Taylor and Sims [8] develop libraries, frameworks and architectures, rather than one model for a single culture; their analytical approach is around modeling and blending physical and cognitive behaviors to deliver cross-cultural training via interactive avatars. The researchers [8] base the modeling framework on ethnographies, cultural behavior theories and cultural studies, concluding, "We believe that deep models of cultural behavior present the best opportunity for building realistic models for effective cross-cultural training."

Okita, Bailenson, and Schwartz [9] (as cited by Lindgren [10]) conduct a virtual reality study and find that people learn more when they believe that a real person, rather than a computer program is controlling the virtual character with whom they are interacting. Taylor and Sims [8] also acknowledge that “...language understanding systems are still not generally robust enough to conduct open-ended conversations with computer-based characters.” Finally, the research [8] still is entirely theoretical; in the context of training it has not yet been applied.

\section{Perspective-taking to Build Intercultural Sensitivity}

Bhawuk, Sakuda, and Munusamy [11] analyze selected learning theories, including the concepts of single-, dou- 
ble- and triple-loop learning, along with intercultural expertise development models. Ultimately, the researchers [11] offer a no-tech intercultural sensitivity learning model based on a video metaphor of pausing; rewinding; forwarding; recording and playing when seeking to gain intercultural sensitivity. A key point is that perspectivetaking is essential, but "...perspective-taking is only possible if the individual's own cultural (stable) perspectives are paused” [11].

Citing Tan \& Yong [12], Bhawuk et al [11] acknowledge that cultural intelligence and intercultural sensitivity can be learned via experiential learning and role-play. Their [11] framework seems excellent; I am simply left eager to associate actual role-plays with it, which they do not do. (I begin to do so in "The Research Design" section.)

\section{E. Virtual Environments’ Perspective-taking Affordances}

Lindgren [10] agrees that perspective-taking is essential to learning, and suggests that what he refers to as virtual environments (VEs) enable a literal perspective-taking that has not been possible prior. The researcher [10] conducts two studies, one involving subjects, wearing a headmounted camera to simulate a first-person perspective, and a second, featuring a training module occurring in a $\mathrm{VE}$, which provides a first-person perspective on "coldrolling” in a steel manufacturing plant. VEs, Lindgren [10] suggests, also highlight a social aspect of perspectivetaking, including multiple perspectives:

VEs can put people in new social contexts that force them to confront the ideas and viewpoints of individuals with different background and experiences.... Participating in a controlled and shared virtual space may make it easier for people to construct a more objective account of their activity. It may also be the case that using a VE with social qualities highlights the importance of multiple perspectives more so than offline activities.

Lindgren's [10] studies come closest to what I am proposing in that he is writing about perspective-taking in connection with learning in a virtual environment, but the studies he does are, by design, canned, rather than in realtime.

Lindgren's [10] research inspires me to keep thinking of how to design virtual world learning modules on cultural intelligence, so that participants can engage in perspective-taking, but in, e.g., Second Life, rather than in a prefabricated simulation; based on the range of literature that I review here, my hypothesis is that cultural intelligence learning is more powerful when one is at least socially immersed, if not physically present, with another real human being(s) during cultural perspective-taking.

It might seem natively obvious, and is supported by Crowne's [3] research, that historically, physical immersion has been the gold standard for cultural intelligence learning, but it remains unaffordable on a massive scale for large, global companies. Since my proposed design allows for non-verbal cues via avatars' body language albeit less subtle than real-life peoples' - and since Second Life allows for real-time in-world voice communications, the interventions I describe in "Research Design" enable learning and immersion beyond standard e-mail, instant-messaging and teleconferences. Also, videoconferencing, while it reveals body language, is expensive and difficult to scale; arguably, Virtual World environments involve less vulnerability than videoconferencing. I qualify vulnerability further:

Action learning through Second Life is a great idea, if designed with the right research question in mind. My primary question is: "What can cultural intelligence learning in Second Life achieve or advance that cultural intelligence learning in real-life cannot as readily?" One hypothesis, which I wish to test repeatedly, is that people do not mind making mistakes in Second Life the way they do in real-life; they learn while feeling less vulnerable than in real-life.

Since people learn more from real people, why not take advantage of the social immersion affordance of Second Life, rather than designing over-engineered, yet still inadequate, smart agents or games for teaching cultural intelligence? Finally, in terms of enabling vivid perspectivetaking, I can imagine building a giant, horizontal taperecorder in Second Life, where learners-as-avatars could stand on the Pause button during the first phase of their intercultural-sensitivity development and so on. Further, for example, Australian, Chinese, U.S.-Anglo and other learners could use the web to study native dress and negotiation styles of a culture, and then enter Second Life and role-play being of the culture.

\section{THE RESEARCH DESIGN}

My research aim is to demonstrate that businesspeople can gain a degree of cultural intelligence, i.e., effectiveness and openness to working with people from other countries, through experiential and action learning in a virtual world such as Second Life - that such an intervention can be the next best thing to being there, and in a number of cases, even more effective than being there. Relatively little is written on how one learns or gains cultural intelligence, since, historically, the world-class way has been via direct experience in the cultures, and perhaps, a degree of cultural intelligence is innate, which makes teaching it further challenging. With the high volume of businesspeople, needing cultural intelligence in today's global economy, however, large-scale, physical immersion simply is unaffordable. In response to the affordability concern, and to my theory that making mistakes in a virtual-world setting is more comfortable and less emotionally and politically costly than making them in a realworld environment, I have co-designed two interventions: piloted in December, 2009 and January, 2010.

Fig. 1 shows the multilingual welcome-sign that appears for both sessions in the main room of the IBM Learning Commons in Second Life. (One of the participants, also a sponsor, is Scottish and I include three versions of Welcome in his native language, since I am able to find three versions on the web.) Unfortunately, not all of the native-language greetings are currently available on the web in the language's alphabet; nonetheless, the welcome-sign is a powerful reminder of the geographic diversity of the participants in real-life, and includes additional languages, to accommodate future participants' native languages.

Positive, online, anonymous participant feedback from both pilots includes: 75 percent of the respondents agree with the statement, "I felt more free to interact as an avatar than I would have felt face-to-face with colleagues.” The implications of their comfort are significant: Naturally, 


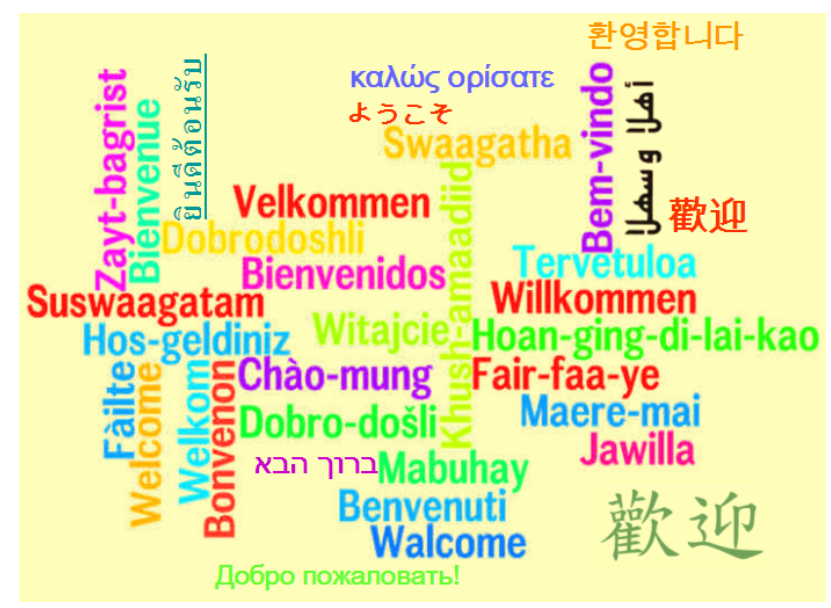

Figure 1. Multilingual welcome sign that participants see upon their arrival in the IBM Learning Commons in Second Life.

when we feel less self-conscious [about revealing a lack of cross-cultural knowledge, or any lack of knowledge], we are more receptive to learning.

Based on other participant feedback and my own observations, the first pilot is ready for formal rollout while the second one requires a bit more format-refinement, e.g., the interviews, I have concluded, should take place by voice, rather than text-chat, as participants report that it feels too disjointed to try to have a meaningful exchange in $10 \mathrm{~min}-$ utes each via text-chat.

I have proposed to my management to formalize the first pilot as a learning activity embedded in Succeeding at IBM, an orientation program provided to all new IBMers worldwide. Based on one of the sponsor's own participation in the first pilot, and on the online, anonymous feedback from participants, they have concurred. In addition, it could be a stand-alone Cultural Intelligence offering we roll out at IBM in 2010, with all employees being eligible to enroll in it, depending on facilitator availability.

Following are details of the pilots: Each of them includes 10 IBM managers from 10 countries. The managers are selected based on their geographic diversity and their prior comfort with Virtual World environments; for the pilots, I did not want to add the learning challenge of being new to Second Life. Of course, in an IBM-wide rollout, that would be a challenge to deal with, which is acknowledge further in "The Research Design Discussion” section. The co-facilitators, Keiko Watanabe and I, share an IBM professional facilitator heritage, in AP and globally, and Amy Groves is a Second Life expert with whom I work in IBM's Center for Advanced Learning.

The managers first meet in the IBM Learning Commons in Second Life on December $15^{\text {th }}$ for a 60 -minute orientation to the IBM Learning Commons; on December $17^{\text {th }}$ for the first pilot - a 60-minute intervention; and on January $19^{\text {th }}$ for the second pilot, also a 60-minute intervention. Before arriving for their orientation, they register in the Country Navigator and complete the virtual tour of the web site; the Country Navigator is a site by TMA (http://www.tmaworld.com/); it is a premier example of the encyclopedic, self-service web sites referred to in the abstract; as an aid for the pilots, it is excellent, though, I believe, insufficient without the live, multi-person, accompanying Virtual-World-based experience.
During the first pilot, they explain how they came by their first and surnames or their avatar names, and offer one thought each on: "What makes you most proud of your culture?" and, "What would you like others to understand better about your culture?”

After discussion in plenary, they brainstorm on how to apply their learning to their leadership roles; reflect for five minutes individually; and complete an anonymous survey designed to enable their self-assessment of learning gain and satisfaction. In addition, afterwards, they participate in interviews with Stanford University visiting scholars, Dr. Petra Bosch and Dr. Anu Sivunen, where they discuss their perspectives on their experience; interview transcriptions are not yet complete.

Two weeks ahead of their re-entering the IBM Learning Commons for the second pilot, the co-facilitators group them with another manager to interview, and to interview them; they include American, Brazilian, Canadian, Chinese, Belgian, Danish, French, German, Indian and Italian managers. Drawing from the Country Navigator, we provide country-culture tip-sheets, according to the pairing, e.g., Belgium and India. Each participant uses the tipsheets to study the country that he or she is representing as well as that of his or her partner.

After each pair conducts its interviews, the members of the pair text-chat about what went well and what could have gone even better; and then we discuss the experience in plenary via text-chat; and then they brainstorm by textchat on applying the learning to their jobs; and then they reflect individually for three minutes; and finally, complete a web-based, anonymous survey designed to enable their self-assessment of learning gain and satisfaction. I compared the surveys from both sessions to determine the sessions' usefulness and conclude that a bit more tweaking is needed for the second pilot, in terms of format, i.e., more voice and less text-chat will be optimal in the revised module.

In Fig. 2, they are debriefing.

\section{RESEARCH DESIGN DISCUSSION}

My literature review of doctoral dissertations and peerreviewed journal articles reveals that apparently, no scholar yet has written about or facilitated action learning intentionally on cultural intelligence in a live, Virtual World setting, and so our facilitation is the most direct way to test the assumption that such learning is possible

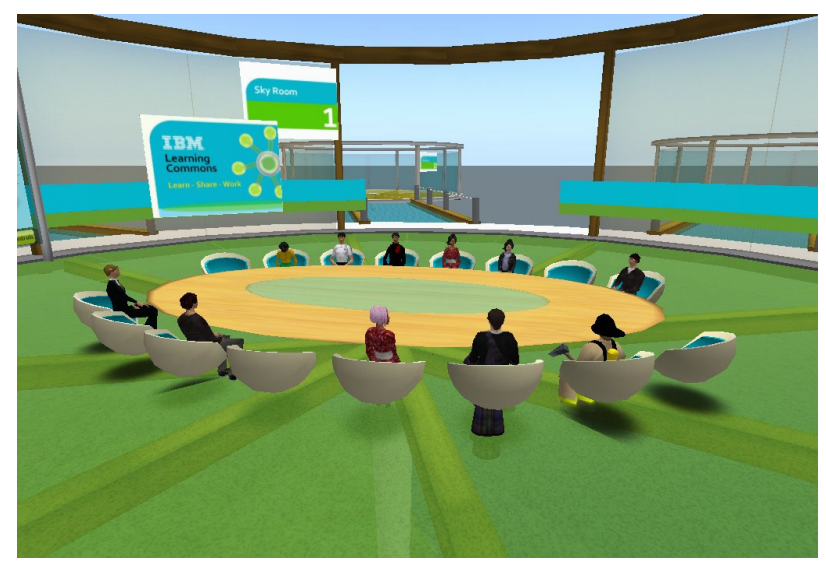

Figure 2. Sky Room 1 of the IBM Learning Commons in Second Life. 
via, e.g., Second Life. So far, the outcomes point to self-reporting of learning gain and increased openness to working with businesspeople from other countries.

Still, there are a number of limitations to this study. Although the interventions are repeatable and should contain the same process each time, internal validity should not result, since the participants are going to vary, and one participant's reaction to the treatment should vary from another's. The other reason it is going to lack internal validity is that by design, I do not have a control group, and so it is going to be difficult to know definitively whether or not the intervention creates the change, or if some other, unobserved factor does.

The 2010 interventions ought to yield external validity for the following populations, since we are including these populations in any rollout: new IBMers; future global assignees; and all employees who are pursuing cultural intelligence.

The sample size is small by design and so probably, we are going to need to do a broader series of interventions before we can be conclusive about their effectiveness. The sample size is 12 because they are 60 -minute sessions and we need to ensure sufficient time for sharing about their cultures and role-playing, along with the debriefing and reflection. Another consideration is Virtual World technology's current, relative instability; today, the more avatars in one virtual place at one time, the more systemcrashes tend to occur.

In terms of selection bias, the sample selection is not representative of new IBMers; future global assignees; non-managers; and Virtual World neophytes; all of them are omitted variables. While a number of the managers have been global assignees in the past, and all of them have been employees prior to being promoted, we need to test the interventions with future assignees; non-managers; and Second Life beginners as well.

That there is not yet a universally-accepted means of measuring cultural intelligence is this research's biggest challenge. Still, I believe in the value of this research design; whether or not we have a precise way of measuring cultural intelligence, I believe we can rely on participants' self-assessment on whether or not their openness to working with businesspeople from other countries increases as a result of the interventions, and their embarrassment at potential cultural gaffes decreases compared to how they imagine they would feel if the same faux pas occurred in real life.

Purposely, I have designed a series of progressively more challenging interventions. By running these pilots, we have been able to ask participants to report on whether or not the Virtual World setting makes them feel more comfortable with making mistakes that they would not be face to face, which they report it does. Comfort and lack of self-consciousness leads to openness at the prospect of working ongoingly with businesspeople from other cultures.

The other biggest challenge is the interventions' brevity. We require less than four hours' time-investment, and yet wish for a consistently measurable learning gain.

The research design's success also depends on participants sharing openly on their cultures - so far, that is happening; preparing their pre-work sufficiently - to date, that is occurring, too; and providing genuine, constructive feedback to their peers on what is believable and what could be improved in their role-plays - by design, we cannot know that fully, since we have opted not to install recorders, to respect learners' privacy, i.e., just as we typically do in face-to-face interventions, when participants form pairs, triads or small groups and go to breakout rooms for an exercise. Based on text-chat output during the plenary debrief, however, we can infer to a degree that genuine, constructive feedback is happening. With all of these challenges in mind, my early experiments still lead me to believe that the possibilities for cultural intelligence learning while in Virtual World environments are vast. I am excited to be among the trail blazers in this realm!

\section{ACKNOWLEDGMENT}

S.E.S. thanks Laura Potts-Sprague and Gordon Fuller for their sponsorship; Amy Groves for her building expertise, design input and co-facilitation; Keiko Watanabe for her design input and co-facilitation; and Jonah Liebert, Steve Jones, Laura Solomon, Dr. Cheryl Toth, Trena Minudri; Dr. Janis Morariu; and Dr. Victoria Marsick for their design input.

\section{REFERENCES}

[1] Triandis, H.C. (2008). Foreword: Cultural Intelligence. In Ang, S., \& Van Dyne, L. (Eds.), Handbook of Cultural Intelligence: Theory, Measurement, and Applications (pp. xi-xiii). Armonk, NY: M.E. Sharpe.

[2] Gelfand, M.J., Imai, L., \& Fehr, R. (2008). Thinking Intelligently About Cultural Intelligence: The Road Ahead. In Ang, S., \& Van Dyne, L. (Eds.), Handbook of Cultural Intelligence: Theory, Measurement, and Applications (pp. 375-387). Armonk, NY: M.E. Sharpe.

[3] Crowne, K.A. (2008). What leads to cultural intelligence? Business Horizons, Vol. 51, 391-399. doi:10.1016/j.bushor.2008. $\underline{03.010}$

[4] Adler, N.J., \& Gunderson, A. (2007). "Managing Multicultural Teams” In Adler, N.J., \& Gundersen, A. (Eds.), International Dimensions of Organizational Behavior (pp. 126-156). Florence, KY: South-Western Educational Publishing.

[5] Fiedler, F.E., Meuwese, W.A.T., \& Oonk, S. "Performance on Laboratory Tasks Requiring Group Creativity,” Acta Psychological, vol. 18 (1961), pp. 110-119. Cited in Adler, N.J., \& Gunderson, A. (2007). "Managing Multicultural Teams” In Adler, N.J., \& Gundersen, A. (Eds.), International Dimensions of Organizational Behavior (pp. 126-156). Florence, KY: South-Western Educational Publishing

[6] Ang, S., Van Dyne, L. Yee, N.K., Koh, C. (2004). The measurement of cultural intelligence. Paper presented at the Annual Meeting of the Academy of Management, New Orleans, LA.

[7] Wagner, C., \& Ip, R.K.F (2009). Action Learning with Second Life - A Pilot Study. Journal of Information Systems Education, Vol. 20(2), 249-258.

[8] Taylor, G., \& Sims, E. (2009). Developing Believable Interactive Cultural Characters for Cross-Cultural Training. In Ozok, A.A. and Zaphiris, P. (Eds.), Online Communities: Lecture Notes in Computer Science (pp. 282-291). Berlin: Springer-Verlag. doi:10.1007/978-3-642-02774-1_31

[9] Okita, S. Y., Bailenson, J., \& Schwartz, D. L. (2007). The mere belief of social interaction improves learning. Proceedings of the Twenty-ninth Meeting of the Cognitive Science Society. Nashville, TN. Cited in Lindgren, R. (2009). Perspective-based Learning in Virtual Environments. Proquest LLC (UMI No. 3364505).

[10] Lindgren, R. (2009). Perspective-based Learning in Virtual Environments. Proquest LLC (UMI No. 3364505).

[11] Bhawuk, D.PS., Sakuda, K.H., \& Munusamy, V.P. (2008). Intercultural Competence Development and Triple-Loop Cultural Learning: Toward a Theory of Intercultural Sensitivity. In Ang, S., \& Van Dyne, L. (Eds.), Handbook of Cultural Intelligence: The- 


\section{GAINING CUltural INTELLIGENCE THROUGH SECOND LIFE LEARNING INTERVENTIONS}

ory, Measurement, and Applications (pp. 342-355). Armonk, NY: M.E. Sharpe.

[12] Tan, J.S., \& Yong, R. (2003). Training and developing cultural intelligence. In P.C. Earley \& S. Ang (Eds.), Cultural intelligence (pp. 258-303). Palo Alto, CA: Stanford University Press. Cited in Bhawuk, D.PS., Sakuda, K.H., \& Munusamy, V.P. (2008). Intercultural Competence Development and Triple-Loop Cultural Learning: Toward a Theory of Intercultural Sensitivity. In Ang, S., \& Van Dyne, L. (Eds.), Handbook of Cultural Intelligence: Theory, Measurement, and Applications (pp. 342-355). Armonk, NY: M.E. Sharpe.

\section{AUTHOR}

Sarah E. Siegel is with IBM's Center for Learning and Development, Armonk, NY 10504 USA (e-mail: ssiegel@us.ibm.com) and an Organization \& Leadership Master student at Columbia University's Teachers College, New York, NY 10027 USA (e-mail: ses2165@columbia.edu).

This work was supported by IBM's Center for Learning and Development and IBM's Center for Advanced Learning. Manuscript received June $15^{\text {th }} 2010$. Published as resubmitted by the author July $29^{\text {th }}, 2010$. 